\title{
Relapsed, refractory peripheral T-cell lymphoma not otherwise specified successfully treated with allogeneic haematopoietic stem cell transplantation
}

\author{
L V Gooneratne ${ }^{1,2}$, V N U Gamage ${ }^{1,3}$, M V C De Silva', P Karunanayake ${ }^{2,4}$ \\ Ceylon Medical Journal 2018; 63: 78-79 \\ DOI: http://doi.org/10.4038/cmj.v63i2.8672
}

\section{Introduction}

Peripheral T-cell lymphoma (PTCL) is a rare haematological malignancy accounting for $3.7 \%$ of all lymphomas and $10-15 \%$ of all non-Hodgkin lymphomas (NHL) [1,2]. Peripheral T-cell lymphoma not otherwise specified (PTCL, NOS) is the commonest subtype of PTCL. However, its' prevalence is lower in Asia than in the West [3]. Only 11 cases of T-cell lymphoma, NOS were reported in Sri Lanka in 2010 [4]. However, PTCL, NOS subtype is not mentioned in this report. PTCL, NOS is a highly aggressive lymphoma notorious for chemo-refractoriness and frequent relapses. High-dose chemotherapy with autologous stem cell transplantation is incorporated in to primary therapy for young fit patients but remains ineffective for most and has not been tested in a randomized study [5]. Allogeneic haematopoietic stem cell transplantation (allo-HSCT) may offer a potential cure for these patients though the optimal type and timing of transplantation remain to be defined [6].

We report a case of relapsed chemo-refractory PTCL, NOS in a young Sri Lankan man who responded to an allo-HSCT performed for the first time in Sri Lanka.

\section{Case report}

A 37-year old previously healthy man from Sabaragamuwa province in Sri Lanka presented with a productive cough and progressive loss of appetite and weight over 2 months. Examination showed generalized lymphadenopathy without any skin lesions or hepatosplenomegaly.

Histology of cervical lymph node biopsy showed, infiltration by intermediate sized lymphocytes with irregular nuclei, open chromatin and nucleoli with moderate cytoplasm. Immunohistochemistry showed CD4, $\mathrm{CD} 5, \mathrm{CD} 3$ and $\mathrm{CD} 20$ positivity and negative for $\mathrm{CD} 7$, CD8, CD10, CD15, CD23, CD30, CD138, ALK1, PAX5, PD-1 and CXCL13. Ki67 was 90\%. A few cells resembling Reed Sternberg (RS) cells positive for CD30, PAX5, Epstein-Bar virus were present. Conclusion was PTCL, NOS with CD20 expression. CD20, a marker of B-cell lineage, is an unusual finding in PTCL, NOS as reported in the literature. Haematological and biochemical investigations were normal. PET/CT scan revealed hypermetabolic lymphadenopathy above and below the diaphragm including bilateral cervical, supraclavicular, axillary, hilar, mediastinal, gastrohepatic and gastrosplenic regions and involvement of lungs. Final diagnosis was PTCL, NOS stage IV.

Following 6 cycles of cyclophosphamide, doxorubicin, vincristine and prednisolone (CHOP) chemotherapy he went in to remission by PET/CT. Within 2 months he relapsed with generalized lymphadenopathy and constitutional symptoms. Relapsed PTCL, NOS was confirmed on a repeat lymph node biopsy. Salvage chemotherapy with gemcitabine, cisplatin and dexamethasone was given without a significant response. PET/CT scan confirmed rapidly progressive disease post 2 cycles of chemotherapy.

HLA typing of siblings revealed a 10/10 match with his younger brother. Matched sibling allo-HSCT was done at Asiri Central hospital, Colombo with reduced intensity conditioning (RIC) using Fludarabine, Melphalan and antithymocyte globulin. RIC was selected anticipating disease eradication and achieving a graft versus lymphoma (GVL)

\footnotetext{
'Department of Pathology, Faculty of Medicine, University of Colombo, ${ }^{2}$ Clinical Haematology and Bone Marrow Transplant Unit, Asiri Central Hospital, ${ }^{3}$ Department of Haematology, Teaching Hospital Karapitiya, ${ }^{4}$ Department of Clinical Medicine, Faculty of Medicine, University of Colombo, Sri Lanka.
}

Correspondence: VNUG, e-mail: <nuwancw84@gmail.com>. Received 01 May 2018 and revised version accepted 18 May 2018. 
effect. Cyclosporine was given for graft versus host disease (GVHD) prophylaxis. Peripheral blood stem cells from his brother $\left(\right.$ dose $=9 \times 10^{6} / \mathrm{kg}$ CD34+ cells $)$ were infused on day 0 . It was well tolerated with only grade 2 mucositis. Neutrophil and platelet engraftment was seen on post-transplant day +9 and +10 respectively. Chimerism testing on days $+15,+60,+98$ revealed $>98 \%$ donor DNA.

In the absence of graft versus host disease, cyclosporine was discontinued after day+100. PET/CT scan 4 months' post HSCT showed a complete metabolic response. He is not on any medication and remains well 7 months after HSCT.

\section{Discussion}

PTCL, NOS is diagnosed when other specific entities of mature T-cell lymphomas have been excluded [7]. It is a highly aggressive disease with a 5-year overall survival of 20-30\% and poor response to therapy [7]. Median age of presentation is 60-years. Male predominance. Most present with peripheral lymphadenopathy. Extranodal involvement is common in skin and gastro-intestinal tract. Many, like our patient, present in advanced stages. PTCL, NOS has heterogeneous morphological, immunohistochemistry and molecular features [3]. Reed Sternberg cells are seen in some with Epstein-Bar virus positivity, as in our patient. Cases of PTCL with Epstein-Bar virus positive Reed Sternberg like cells are uncommon [8]. As in our patient, CD20 positivity in PTCL, NOS is also rarely reported and is associated with a more aggressive clinical course [9].

CHOP is the standard first line treatment for primary disease. Consolidation with auto-HSCT may provide a survival benefit, however limited by early relapses and frequent chemo-refractoriness [3]. Aberrant CD20 expression may need further evaluation for Rituximab use.

Relapsed/refractory cases have several options including combination chemotherapy, autologous or allogeneic HSCT, novel therapies including monoclonal antibodies, immuneconjugates, histone deacetylase inhibitors and anti-folates [3].

Reported cases of PTCL, NOS treated with allo-HSCT are extremely rare and it's been suggested that outcomes are better if performed early in relapse [3], as was done in our patient.

\section{Conclusion}

This report adds to the literature the role of alloHSCT in PTCL, NOS and highlights the importance of timely provision of this treatment modality to suitable patients.

\section{Conflicts of interest}

Authors declare that they have no conflicts of interest.

\section{References}

1. Dearden C, Johnson R, Pettengell R, et al. Guidelines for the management of mature T cell and NK cell neoplasms excluding cutaneous T cell lymphoma. Updated August 2013. British Committee for Standards in Haematology, 2013. http://www.b-s-h.org.uk/guidelines.

2. d'Amore F, Gaulard P, Trumper L, et al. Peripheral T-Cell Lymphomas: ESMO Clinical Practice Guidelines for diagnosis, treatment and followup. Ann Oncol 2015; 26: $108-15$.

3. Broccoli A, Zinzani PL. Peripheral T-cell lymphoma, not otherwise specified. Blood 2017; 129: 1103-12.

4. Cancer Incidence Data Sri Lanka 2010, National Cancer Control Programme. http://nccp.health.gov.lk/images/ PDF_PUBLICATIONS/Cancer_Incidence_Data_2010.pdf

5. Al-Zahrani M, Savage KJ. Peripheral T-Cell Lymphoma, Not Otherwise Specified: A Review of Current Disease Understanding and Therapeutic Approaches. Hematol Oncol Clin North Am 2017; 31(2): 189-207.

6. Wei J, Xu J, CaoY, Zhou J, Zhang Y. Allogeneic Stem-Cell Transplantation for Peripheral T-Cell Lymphoma: A Systemic Review and Meta-Analysis. Acta Haematol 2015; 133(2): 136-44.

7. Pileri S A, Weisenburger DD, Sng I, et al. Peripheral T-cell lymphoma, not otherwise specified. In: Swerdlow SH, Campo E, Harris NL, et al. World Health Organization classification of tumours of haemopoietic and lymphoid tissues, 4thedition. Lyon, France: IARC Press; 2008: 306-8.

8. Kaur P, Levy NB. Peripheral T-cell lymphoma, not otherwise specified with Reed Sternberg like cells. Am J Haematol 2011; 86: 1035.

9. Hu S, He X, Shi Y, et al. Clinical features and prognosis of CD 20 positive Peripheral T-cell lymphoma, not otherwise specified in Chinese Patients: A retrospective single institution analysis. Blood 2015; 126: 5045. 